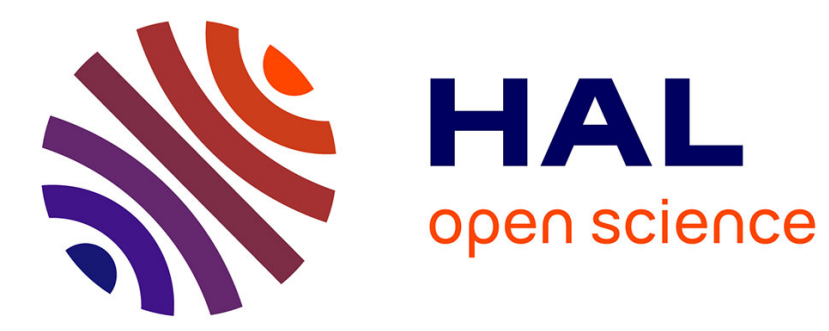

\title{
Experimental characterisation of activated sludge behaviour during mechanical expression
}

M. Raynaud, P. Héritier, J.C. Baudez, Jean Vaxelaire

\section{To cite this version:}

M. Raynaud, P. Héritier, J.C. Baudez, Jean Vaxelaire. Experimental characterisation of activated sludge behaviour during mechanical expression. Process Safety and Environmental Protection, 2010, 88, 8 p. 10.1016/j.psep.2010.02.004 . hal-00506652

\section{HAL Id: hal-00506652 \\ https://hal.science/hal-00506652}

Submitted on 28 Jul 2010

HAL is a multi-disciplinary open access archive for the deposit and dissemination of scientific research documents, whether they are published or not. The documents may come from teaching and research institutions in France or abroad, or from public or private research centers.
L'archive ouverte pluridisciplinaire HAL, est destinée au dépôt et à la diffusion de documents scientifiques de niveau recherche, publiés ou non, émanant des établissements d'enseignement et de recherche français ou étrangers, des laboratoires publics ou privés. 


\title{
Experimental characterisation of activated sludge
}

\section{behaviour during mechanical expression}

\author{
M. Raynaud ${ }^{1,2}$, P. Heritier ${ }^{1}$, J-C. Baudez ${ }^{1 *}$, and J. Vaxelaire $^{2}$ \\ ${ }^{1}$ CEMAGREF, Domaine des Palaquins, 03150 Montoldre, France \\ ${ }^{2}$ LaTEP, rue Jules Ferry, 64075 Pau cedex, France
}

\begin{abstract}
Even after mechanical dewatering, activated sludge contains a large amount of water. Due to its biological nature, composition and also the type of treatment it comes from, this material is usually highly compressible and known to be difficult to dewater. In the present work various tests are proposed to try to highlight some aspects which could explain the poor dewaterability of activated sludge. Experiments of expression were carried out in a Filtration-Compression Cell for semi-solid samples of sludge. Operating conditions (intensity of the applied pressure, initial thickness of sludge sample) and the composition of the sludge (ionic strength by adding monovalent salt) were modified for the different series of experiments. It was observed that the removal of water from the sludge depended significantly on the pressure gradient throughout the sludge cake. However, the development of a dense layer of cake at the interface cake/filter medium seems to generate a pressure gradient non-uniformly distributed throughout the thickness of the cake. This phenomenon should control in part the efficiency of dewatering. This study also discusses the link between sludge dewaterability and cake relaxation on the basic of an osmotic effect within the flocculated matrix, which tends to resist against compression or deformation. This effect was reduced when both the applied pressure and the time increased. Finally, the activated sludge dewatering during expression stage depends on both the formation *Corresponding author: Tel: +(33)-(0)4-70-47-74-10/Fax: +(33)-(0)4-70-47-74-11

E-mail: jean-christophe.baudez@cemagref.fr
\end{abstract}


of a dense layer of cake at the interface cake/filter medium and an osmotic effect.

Keywords: activated sludge; dewatering; osmotic effect; dense layer of cake; relaxation.

\section{Introduction}

Wastewater treatment plants produce a large amount of residue. European activated sludge production approximately reaches twelve millions tons of dry matter per year. Consequently, a reduction of sludge volume is generally required before handling and disposal. Due to their relatively low energy consumption, mechanical processes, such filter presses or centrifuges, are usually used to carry out the dewatering. However, the final water content of the sludge remains generally high.

The ability of sludge to dewater largely depends on its nature, its composition and also on the type of treatment it comes from. Many researches have been carried out to explain how these different aspects could impact the dewatering efficiency. Some authors highlighted correlations between sludge characteristic parameters (concentration and composition of EPS, concentration of multivalent cations, hydrophobicity...) and the ability to dewater evaluated by parameters such as CST (Capillary Suction Time) or SRF (Specific Resistance of Filtration) (Karr and Keinath, 1978; Wu et al., 1982; Novak et al., 1998; Mikkelsen and Keiding, 2002). However, those parameters are not appropriated to fully characterise sludge dewatering. Indeed, different works have highlighted the weakness of the CST method (Herwijn, 1996; Smiles, 1998). This method must be considered more as an empirical tool for engineering practises to optimise flocculation than as a test that allows a careful analysis of dewatering devices. In parallel, SRF is not sufficient to fully describe the behaviour of the sludge in the conventional dewatering processes, which usually work in both filtration and expression stages.

At laboratory scale, both stages of the dewatering can be well characterized by experiments carried out in a Filtration-Compression Cell (FCC).

The first one corresponds to the cake formation caused by an accumulation of solid particles on 
the surface of the filter medium. The second one relates to the removal of water by cake squeezing.

A lot of work has been carried out, with different kinds of suspensions on FCC to study both: filtration and expression with varying characteristics parameters, such as the applied pressure. For highly compressible materials, such as activated sludge, Tiller and Kwon (1998) showed that dewatering was not affected by the applied pressure during the filtration stage. Sorensen and Hansen (1993) exhibited that this effect seems due to a thin layer of cake which is formed during the filtration stage at the interface cake/filter medium, resulting in an unexpected high value of the Specific Resistance of Filtration.

During the expression stage, the sludge behaviour was commonly analysed by combined Terzaghi-Voigt rheological models (Chang and Lee, 1998) as initially proposed by Shirato et al.(1986).

In the case of activated sludge, some authors recently suggested that an osmotic effect should be considered to well describe the dewatering process (Keiding and Rasmussen, 2003; Curvers et al., 2009). Indeed, due to its biological nature, activated sludge possesses a complex chemical composition. This system can be represented by a polymeric matrix wherein microbial organisms are embedded (Jorand et al., 1995; Higgins and Novak, 1997). These extracellular polymeric substances (EPS) carry charged functional groups. It has been shown that the sludge dewatering behaviour can be influenced by the osmotic pressure within the flocculated matrix, namely by the presence of charged surface groups (Keiding et al., 2001; Keiding and Rasmussen, 2003). By definition, the osmotic effect is the diffusion of a solvent (mostly water) through a semipermeable membrane (which is permeable to the solvent and impermeable to the solute) owed to solute concentration gradient. The solvent flows from the low concentrated solution to the higher one, due to a difference of chemical potential between both regions. 
The osmosis phenomenon may be opposed by increasing the applied pressure on the region of high solute concentration with respect to that on the low solute concentration region. The pressure required to prevent the passage of water through the semi-permeable membrane is equivalent to the osmotic pressure of the solution. At this time the thermodynamic equilibrium (equality of chemical potentials) between concentrated and diluted regions is reached.

Therefore, when the applied pressure is more consequent than the osmotic pressure, the solvent migrates from the concentrated region to the diluted one.

In the case of activated sludge, there is no real semi-permeable membrane to separate liquid with different concentrations of solute. However, the surface of the charged polymeric network could play this role (Curvers et al., 2009). Free solvent (corresponding to the diluted region) flows into the flocculated matrix (corresponding to the concentrated region) to reach the thermodynamic equilibrium. This osmotic effect could induce a sludge cake relaxation phenomenon.

Different tests, carried out in FCC recording piston displacement over time, are presented in this study to better investigate the sludge cake behaviour. The impact of the applied pressure and the initial sample thickness were analysed during expression stage.

\section{Materials and methods}

\subsection{Sludge}

Sludge was sampled at the inlet of the clarifier of a small wastewater treatment plant located in a rural area in Allier (centre of France). It was first concentrated at approximately $10 \%$ of dry matter by using a system of vacuum filtration. The exact concentration was determined by drying at $105^{\circ} \mathrm{C}$ during $24 \mathrm{~h}$ and weighing. Afterward, the concentrated sludge was diluted with the filtrate at approximately $8 \%$ of dry matter in order to obtain a semi-solid sample. In order to exclusively focus our work on the expression stage. 
To reduce the temporal variability due to the biological activity, the prepared sludge was stored at $4^{\circ} \mathrm{C}$ during $24 \mathrm{~h}$ before carrying out dewatering tests. Such a procedure limits the impact of the biological activity on the sludge behaviour and thus enables an acceptable reliability of the experiments as previously showed by Raynaud et al., (2009). However, the same sludge sample must be used for each series of experiments (in other words for one studied parameter) in order to obtain a good reproducibility of experimental results.

To modify chemical potential distribution within the sludge, salt $(\mathrm{NaCl})$ was added to reach approximately $10 \%$ (in mass) of the dry matter content of sludge. Samples were homogenized under mechanical mixing.

\subsection{Filtration-Compression Cell}

The filtration compression cell was a $0.12 \mathrm{~m}$ depth cylindrical stainless steel chamber with an internal diameter of $0.07 \mathrm{~m}$. A perforated disk was located at the bottom of the cylinder to support the filter medium.

Fisherband 112 (porosity of $16 \mu \mathrm{m}$ ) filter papers were used. These filter papers were well adapted for the FCC tests because only few solids particles passed through.

Piston pressure was applied by pressurized air (ranging between 0 and 7 bar) and was piloted by a regulating system using a controlled valve. The mass of filtrate and the piston displacement were recorded on-line. The filtrate evaporation was also measured in parallel with a second weigh-scale along experiments.

Friction stress between the piston and the chamber wall was measured to approximately 2030 Newton, i.e. 0.05 bar.

The accuracy of the displacement gauge was estimated at $10^{-3} \mathrm{~cm}$ according to manufacture data. 


\subsection{Impact of experimental parameters}

In order to understand what really happened within the Filtration-Compression Cell during expression stage, we performed several tests by varying the following parameters: intensity of the applied pressure, the initial thickness of the sample and the ionic strength by adding monovalent salt.

The results showed the role of these parameters on the dewatering kinetics under both constant pressure and a sudden pressure release.

\section{Results and discussion}

\subsection{Impact of the applied pressure and of the initial sludge thickness on the dewatering}

Several tests were carried out in FCC under constant pressures ranging between 1 and 7 bar. The initial thickness of the sludge samples was same for each experiment.

A second set of tests was conducted for different initial amounts of sludge (height of $1 \mathrm{~cm}$ and 2 $\mathrm{cm})$ at a fixed pressure (6 bar). To easily compare the different results the filtrate mass was scaled by the mass of water contained in the sludge at the beginning of the experiments $\left(\mathrm{m}_{0}\right)$.

As it can be seen on figures 1 and 2, the filtrate mass increases when the applied pressure increases and the initial thickness of sample decreases.

These results could be explained, in part, by the average pressure gradient through the cake. The pressure above the cake is close to the applied pressure and the one at the bottom of the filter medium corresponds to the atmospheric pressure. Consequently, the pressure gradient increases with the applied pressure (for a given initial thickness of sample) and decreases when the initial sample thickness is thicker (for a given pressure). Finally, the efficiency of sludge expression can be improved by increasing the pressure gradient.

However, the monitoring of the average strain of the sludge cake calculated throughout experiments $\left(\left(\frac{h_{0}-h}{h_{0}}\right)\right.$, with $h_{0}$ the initial thickness of sludge and $h$ the sludge thickness during 
dewatering), showed that the sludge behaviour was not homogenous.

Intuitively, the representations in terms of scaled mass or strain were supposed to be both similar because the piston displacement was induced by filtrate removal.

However, when the initial thickness of the sample decreased, the final cake dry matter content increased (figure 2) whereas the final average strain of sludge cake was not affected (figure 3).

Consequently, when the scaled mass of filtrate collected was plotted with regards to the average strain of the cake a non linear behaviour was observed (figure 4).

As the sludge was considered as a two non-compressible phases system, the water volume resulting from the piston displacement should be equal to the volume of filtrate (or mass) collected to insure the mass conservation, and a linear evolution was expected on figure 4.

However, when the scaling of the cake thickness was done with regards to the initial mass of sample (instead of initial thickness) an expected linear evolution was observed (figure 5).

The comparison of those two scaling procedures tends to highlight the fact that the cake strain is not uniform throughout the sample thickness.

This heterogeneity is supposed to be due to a layer of denser cake at the interface cake/filter medium, as already suggested by different authors during filtration tests (Tiller and Green, 1973; Sorensen and Hansen, 1993).

Due to its higher dry matter content, this layer should be more compact than the other part of cake, and exhibit smaller strain under pressure. Consequently, the hypothesis assuming a uniform strain throughout the cake thickness can lead to some significant deviations.

The development of this dense layer of cake seems to control, in part, the kinetic of dewatering. Sorensen and Hansen (1993) and more recently Raynaud et al. (2009) indicated that the kinetic of dewatering was not affected by the applied pressure during filtration stage. This behaviour was explained by the rapid formation, at the interface cake/filter medium, of a thin and low permeable layer of cake. The present study tends to show that the formation of a denser layer 
of cake also occurs during expression, but during this stage the kinetic of dewatering depends on the applied pressure and also of the initial thickness of sludge sample. Due to the presence of a dense layer of cake the pressure gradient throughout the cake is not uniform and the efficiency of the expression seems to be limited when the thickness of the layer is high. The dense layer of cake can be considered as a low straining and low permeable new filter medium, which limits the filtrate flow. The densification of the cake near the interface cake/filter medium should be linked to structural modification of the cake. Sludge is mainly made of polymers which are known to relax when submitted to an external stress. This particular behaviour implies that the material modifies its structure for counter balance the stress effects leading to a relaxation of polymeric materials by stress release. Few authors have highlighted this behaviour on sludge cake (Iwata and Murase, 1993; Christensen and Keiding, 2006). To go further in this analysis, different tests were carried out in FCC releasing the pressure during the expression stage.

\subsection{Relaxation phenomenon}

When the applied pressure was released at the end of an expression test, the piston rised (figure 6): the sludge volume in the FCC tends to increase whereas the solid concentration was the same. This phenomenon can be owed to the sludge cake relaxation.

Similar tests were performed several times under the same experimental conditions to follow the upward displacement of the piston. The results of the series of experiments showed a good reproducibility of the measurement (figure 7). Results of two tests were only plotted to obtain a clear figure. 


\subsubsection{Impact of the initial pressure}

Various step pressure experiments were carried out in the FCC, starting from different applied pressures, to study the cake expansion behaviour. In these tests the pressure was kept under a constant value for a given time. Next, the pressure was released and the upward displacement of the piston was recorded. To better compare the different series of experiments, the upward displacement of the piston (h) was scaled by the piston position just before the pressure release $\left(\mathrm{h}^{*}\right)$, i.e. $\left(\frac{h}{h^{*}}\right)$.

Results obtained from these tests showed that the rise of the piston increased with the initial applied pressure (figure 8).

This behaviour could be partly explained by an osmotic effect and related to the work of Curvers and al. (2009). They notably assessed that the osmotic pressure within sludge is close to 2.44 bars. Consequently, it can be supposed that the piston especially rises when the applied pressure is higher than the osmotic pressure.

Due to osmotic effect, water tends to flow into the flocculated matrix when the pressure on the piston is released.

To overcome this osmosis phenomenon, the applied pressure on the solid network of sludge must be higher than the osmotic pressure.

This water flux $\left(J_{W}\right)$ throughout the flocculated matrix could be, in theory, expressed in function of the pressure gradient (see Appendix):

$$
J_{w}=K_{w}(\Delta P-\pi)
$$

with $\Delta P$ the difference between the effective solid pressure $\left(P_{s}\right)$ and the liquid pressure $\left(P_{l}\right)$, and $K_{w}$ the apparent permeability of the flocculated matrix.

Equation (1) is quite difficult to apply on our experiments, indeed it needs to measure the pressure distribution throughout the filter cake over the expression test (see Appendix). Besides, 
the permeability should not be constant, and depend on both the effective solid pressure and the sludge composition. However, according to equation (1), it can be seen that when $\Delta P$ is higher than the osmotic pressure, water is expressed from the flocculated network to the interfloc region and next from the cake to the outside of the system. During expression of the filter cake, the applied pressure is largely transmitted to the solid network and $\Delta P$ tends progressively to increase. The expression process is mainly controlled by the applied pressure as long as $\Delta P$ is high compare to $\pi$. Due to the expression of water to the interfloc region, the mole fraction of water inside the flocculated network tends to decrease with time, leading to a decrease of the water chemical potential.

Consequently, when the magnitude of $\Delta P$ and $\pi$ is comparable, both pressures impact on the dewatering. If the applied pressure is released the water tends to flow into the flocculated matrix, under the action of the osmotic pressure, and the system tends to swell. This relaxation of the flocculated matrix causes the rise of the piston.

Finally, the dewatering kinetic of sludge depends on both the average pressure gradient through the cake and the relaxation phenomenon (figure 1 and 8).

The amount of water removed from the flocculated network to the interfloc region, increased when the applied pressure is high. Thus, this phenomenon can partly explain the improvement of the sludge dewatering efficiency.

\subsubsection{Impact of dewatering time}

Step pressure tests were carried out to analyze the evolution of cake relaxation during a full expression stage. The applied pressure was successively maintained constant and released during regular intervals of time (figure 9); and the rise of the piston was measured when the pressure was released. 
The evolution of the piston position can be observed on figure 10. The upward displacement of the piston remains constant during the first part of the expression stage and then becomes smaller and smaller as time increases.

An osmotic effect leading to swelling and stretching phenomena of the flocculated matrix could explain in part this result. Indeed, during dewatering, the osmotic pressure tends to change due to the evolution of the chemical potential in both, the internal and external regions.

Within the flocculated matrix, the chemical potential of water decreases with time due to the expression of water from the inside of flocculated network to the outside.

Moreover, the water located in the interfloc region is removed from the sludge cake, during expression.

At the beginning of the dewatering, the chemical potential of water is approximately constant because the mole fraction of water remains nearly similar. However, the mole fraction of interfloc water tends to decrease with dewatering, leading to a decrease of the chemical potential of water. Consequently, the difference of chemical potential of water between the inside and the outside of the flocculated network becomes lower and the water flux which migrates into the flocculated matrix, due to osmotic effect, reduces when the applied pressure is released.

In terms of electrostatic interactions this decrease of chemical potential on the interfloc region could be caused by a compression of the electrical double layer. Thus, the swelling and stretching phenomena of the matrix tend to be limited.

\subsubsection{Effect of salt addition}

Step pressure tests similar to those presented in section 3.2.2 were conducted again but with an addition of salt $(\mathrm{NaCl})$.

Three pressure steps were applied at the beginning of the dewatering, where the piston rise is not affected by the time of dewatering. 
As it can be seen in Table 1, piston rising remained constant whatever the time of pressure application was (expected result) but it was lower when $\mathrm{NaCl}$ was added.

Moreover, the final dry matter content of the sludge cake (before pressure release) is slightly higher for the sludge containing salt (49\% instead of $46.5 \%)$.

Addition of salt in the sludge increased the ionic strength of the bulk solution and thus decreased the chemical potential of water in the interfloc region. Consequently, the osmotic pressure was modified, and the amount of water flowing from the outside to the inside of the flocculated matrix was reduced.

Furthermore, the reduction of $\pi$, due to salt addition, enhanced the migration of water from the inside to the outside of the flocculated network and resulted to an higher final dry matter content of the sludge cake under pressure when salt was added.

This last set of experiments confirmed that the osmotic effect resists against compression and tends to limit the sludge dewatering. However the osmotic effect induced by the difference of ionic stress should not be the only mechanism for sludge relaxation. Other structural components should also be taken under consideration such as the affinity between the water and the network or the reticulation ratio of the polymer chains (Legrand, 1997; Curvers and al. 2009).

\subsubsection{Relation with the dense layer of cake}

Several tests were carried out in FCC for different thicknesses of the initial sample. All experiments were conducted under a constant pressure during the same time, and the piston displacement was measured when the applied pressure was suddenly released.

Results reported in table 2 indicate that the rise of the piston remained constant whatever the initial thickness of the sample was. 
The osmotic pressure within sludge seemed to be the same for different initial thicknesses (the water content in the inside and the outside regions of the flocculated matrix was the same). Consequently, the swelling percentage of the sludge cake might remain identical. Due to the increase of the initial thickness, the density of flocs in the sample should be more important. Intuitively, the upward displacement of the piston should be higher. However, according to the data reported in the table 2 the swelling percentage of cake seemed to depend on the initial thickness of sample. This result could be explained by the fact that the sludge cake was not homogenous before swelling phenomenon occurred.

Finally, it can be assumed that the dense layer of cake was not strained during expression. With a cake thickness similar to the dense layer, the piston should be not rise (when the applied pressure released). Consequently, the observation of the relaxation phenomenon can be perturbed by the development of the dense layer of cake on the filter medium during expression stage.

\section{Conclusion}

The kinetic of expression of semi-solid activated sludge samples was studied from experiments carried out in Filtration-Compression Cell. Tests were conducted under various operating conditions (intensity of the applied pressure, initial thickness of sludge sample) and for two different compositions of the sludge (modify by adding monovalent salt). It was observed that the removal of water from the sludge depended significantly on the pressure gradient within the cake. However, this pressure gradient was not uniformly distributed throughout the cake thickness due to the development of a low-permeable and low-straining layer of cake at the interface cake/filter medium. As it was already shown by other authors for filtration stage, the development of this layer tends to impact significantly the sludge dewatering during expression stage.

This study also pointed out the impact of an osmotic effect on the dewatering behaviour. This aspect was essentially highlighted from a study of sludge cake relaxation based on pressure 
release tests. Finally, sludge dewatering by expression seems to depend on both, the development of a dense layer of cake on the filter medium and on osmotic effect. Further work is needed to better evaluate the impact of both aspects and the implementation of pressure sensors along the cylindrical chamber of the FCC to measure pressure profile throughout the cake seems to be a interesting improvement of experimental set-up in order to reach this goal.

Many parameters relative to the nature of activated sludge (for example: concentration and composition of exopolymers, concentration of multivalent cations, hydrophobicity, surface charges...) should have an impact on both, the development of the dense layer and the osmotic effect. Consequently, further works are needed to better understand the ability of the sludge to squeeze and to express water, during expression stage.

\section{Acknowledgements}

The authors wish to express their sincere gratitude to the French Agency for Environment and Energy Management (ADEME) for the financial support of this study. 


\section{References}

Chang, I.L., and Lee, D.J., 1998, ternary expression stage in biological sludge dewatering. Water Research, 32: 905-914.

Christensen, M. L. and Keiding, K., creep effects in activated sludge filter cakes. Powder Technology, 177(1): 23-33.

Curvers, D., Usher, S. P., Kilcullen, A. R., Scales, P. J., Saveyn, H. and Van der Meeren, P., 2009, the influence of ionic strength and osmotic pressure on the dewatering behaviour of sewage sludge. Chemical Engineering Science, 64(10): 2448-2454.

Herwijn, A.J.M., 1996, fundamental aspects of sludge characterization. PhD thesis, Technische Universiteit Eindhoven.

Higgins, M. J. and Novak, J. T., 1997, characterization of exocellular protein and its role in bioflocculation. Journal of Environmental Engineering, 123(5): 479-85.

Infelta, P. and Graetzel, M., 2006, thermodynamiques : principes et applications. Brownwalker Press.

Iwata, M. and Murase, T., 1993, stress relaxation of expressed material. $6^{\text {th }}$ world filtration congress, Nagoya.

Jorand, F., Zartarian, F., Thomas, F., Block, J., Bottero, J., Villemin, G.., Urbain, V. and Manem, J., 1995, chemical and structural (2D) linkage between bacteria within activated sludge flocs. Water Research, 29(7): 1639-1647.

Karr, P. R. and Keinath, T. M., 1978, influence of particle size on sludge dewatering. Journal Water Pollution Control Federation, 50: 1911-1930.

Keiding, K. and Rasmussen, M. R., 2003, osmotic effects in sludge dewatering. Advances in Environmental Research, 7(3): 641-645.

Keiding, K., Wybrandt, L. and Nielsen P., 2001, remember the water - a comment on EPS colligative properties. Water Science and Technology, 43(6): 17-23. 
Legrand, V., 1997, étude du gonflement et de la flocculation de gels de polyelectrolytes: application à la déshydratation des boues de station d'épuration. PhD thesis, Université Pierre et Marie Curie- Paris VI.

Mikkelsen, L. H. and Keiding, K., 2002, physico-chemical characteristics of full scale sewage sludges with implications to dewatering. Water Research, 36: 2451-2462.

Mulder, M., 1997, Basic Principles of membrane technology. $2^{\text {nd }}$ edition. Kluwer Academic Publishers.

Novak, J. T., Love, N. G, Smith, M. L. and Wheeler E. R., 1998, the effect of cationic salt addition on the settling and dewatering properties of an industrial activated sludge. Water Environment Research, 70: 984-996.

Raynaud, M., Baudez J-C., Heritier, P. and Vaxelaire J., 2009, activated sludge dewatering in Filtration-Compression Cell: deviations in comparison to classical theory. Asia-Pacific Journal of Chemical Engineering. doi 10.1002/apj407

Shirato, M, Murase, T., Iwata, M., and Nakatsuka, S., 1986, the Terzaghi-Voigt combined model for constant-pressure consolidation of filter cakes and homogeneous semi-solid materials. Chemical Engineering Science, 41(12): 3213-3218.

Smiles, D.E., 1998, water flow in filter paper and capillary suction time. Chemical Engineering Science, 35(12): 2211-2218.

Sorensen, P. B. and Hansen, J. A., 1993, extreme solid compressibility in biological sludge dewatering. Water Science and Technology, 28(1): 133-143.

Tiller, F.M. and Green T.C., 1973, the role of porosity in filtration: IX. Skin effect with highly compressible materials. AICHEJ, 19: 1266-1269.

Wu, Y. C., Smith, Ed D. and Novak R., 1982, filterability of activated sludge in response to growth conditions. Journal of Water Pollution Control Federation, 54: 4446-456. 


\section{Appendix}

The permeation flux of water through a membrane can be expressed by (Mulder, 1997):

$$
J_{w}=k_{w} \frac{d \mu_{w}}{d z}
$$

with $\mu_{w}$ the chemical potential, $k_{w}$ the membrane permeability and $z$ the coordinate perpendicular to the transport barrier (membrane or polymeric network in our case).

On the surface of the charged polymeric network, assuming an ideal solution, the chemical potential of water can be calculated by (Infelta and Graetzel, 2006):

$$
\mu_{w}\left(T, P, x_{w}\right)=\mu_{w}^{0}(T)+R T \ln \left(x_{w}\right)+\int_{P}^{P+\pi} V_{m}^{*} d P
$$

where $\mu_{w}^{0}$ is the standard chemical potential of the water, $R$ the universal gas constant, $x_{w}$ the mole fraction of water in the solution, $V_{m}^{*}$ the molar volume of water, $\pi$ the osmotic pressure, $\mathrm{T}$ and $\mathrm{P}$ the temperature and the pressure of the system.

As the chemical potential only depends on both the applied pressure and the molar fraction of water, the gradient in equation (A.1) can be written from the derivation of equation (A.2):

$$
\frac{d \mu_{w}}{d z}=V_{m}^{*} \frac{d P}{d z}+\frac{R T}{x_{w}} \frac{d x_{w}}{d z}
$$

Moreover, according to the van't Hoff equation the osmotic pressure can be written as:

$$
\pi=-\frac{R T}{V_{m}^{*}} \ln \left(x_{w}\right)
$$

Considering equations (A.3) and (A.4), the gradient of the chemical potential can be given by:

$$
\frac{d \mu_{w}}{d z}=V_{m}^{*} \frac{d(P-\pi)}{d z}
$$

Finally, the water flux can be written as:

$$
J_{w}=K_{w} \frac{d(P-\pi)}{d z}
$$


Integrating equation (A.6) on the network thickness, it comes:

$$
J_{w}=K_{w}\left[\left(P_{s}-\pi\right)-\left(P_{l}\right)\right]
$$




\section{Figure captions}

Figure 1: Mass of filtrate collected at various applied pressures versus time for semi-solid sludge (initial thickness of $2 \mathrm{~cm}$ ).

Figure 2: Scaled mass of filtrate collected at various initial thicknesses versus time for semisolid sludge (applied pressure of 6 bar).

Figure 3: Average strain of the sludge cake versus time at various initial thicknesses (applied pressure of 6 bar).

Figure 4: Scaled mass of filtrate collected versus the average strain of the sludge cake (initial thickness of $2 \mathrm{~cm}$ and applied pressure of 6 bar).

Figure 5: Scaled mass of filtrate collected versus the scaling of the cake thickness by the initial mass of sample (initial thickness of $2 \mathrm{~cm}$ and applied pressure of $6 \mathrm{bar}$ ).

Figure 6: Piston displacement versus time.

Figure 7: Test of reproducibility, rise of the piston versus time (initial thickness of $2 \mathrm{~cm}$ and applied pressure of 6 bar; time equal to zero corresponds to the pressure release).

Figure 8: Rise of the piston at different steps of pressure (initial thickness of $2 \mathrm{~cm}$ ).

Figure 9: Evolution of the applied pressure with time.

Figure 10: Rise of the piston versus time at various pressure steps (initial thickness of $2 \mathrm{~cm}$ ). 
Figure 1:

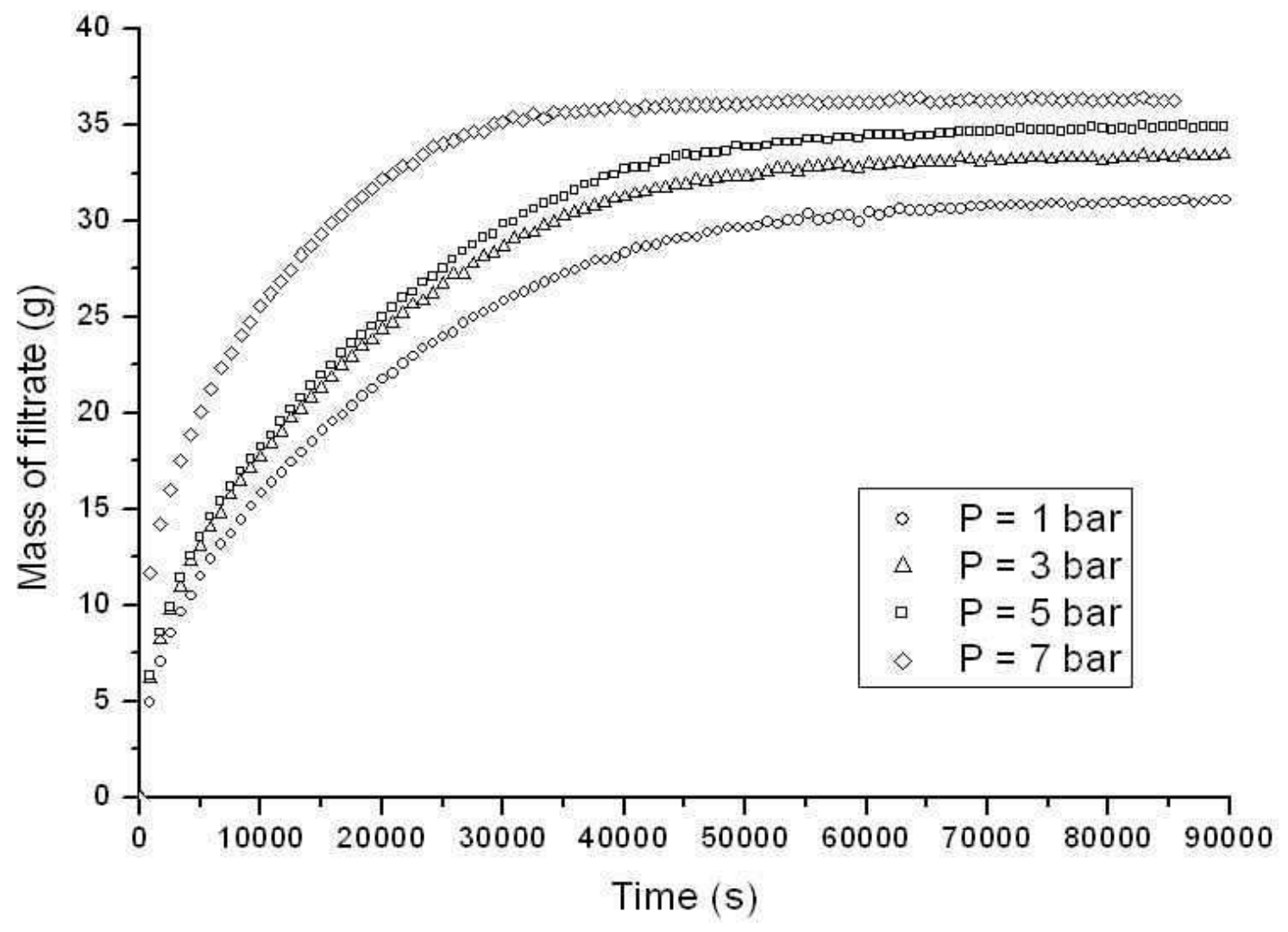


Figure 2:

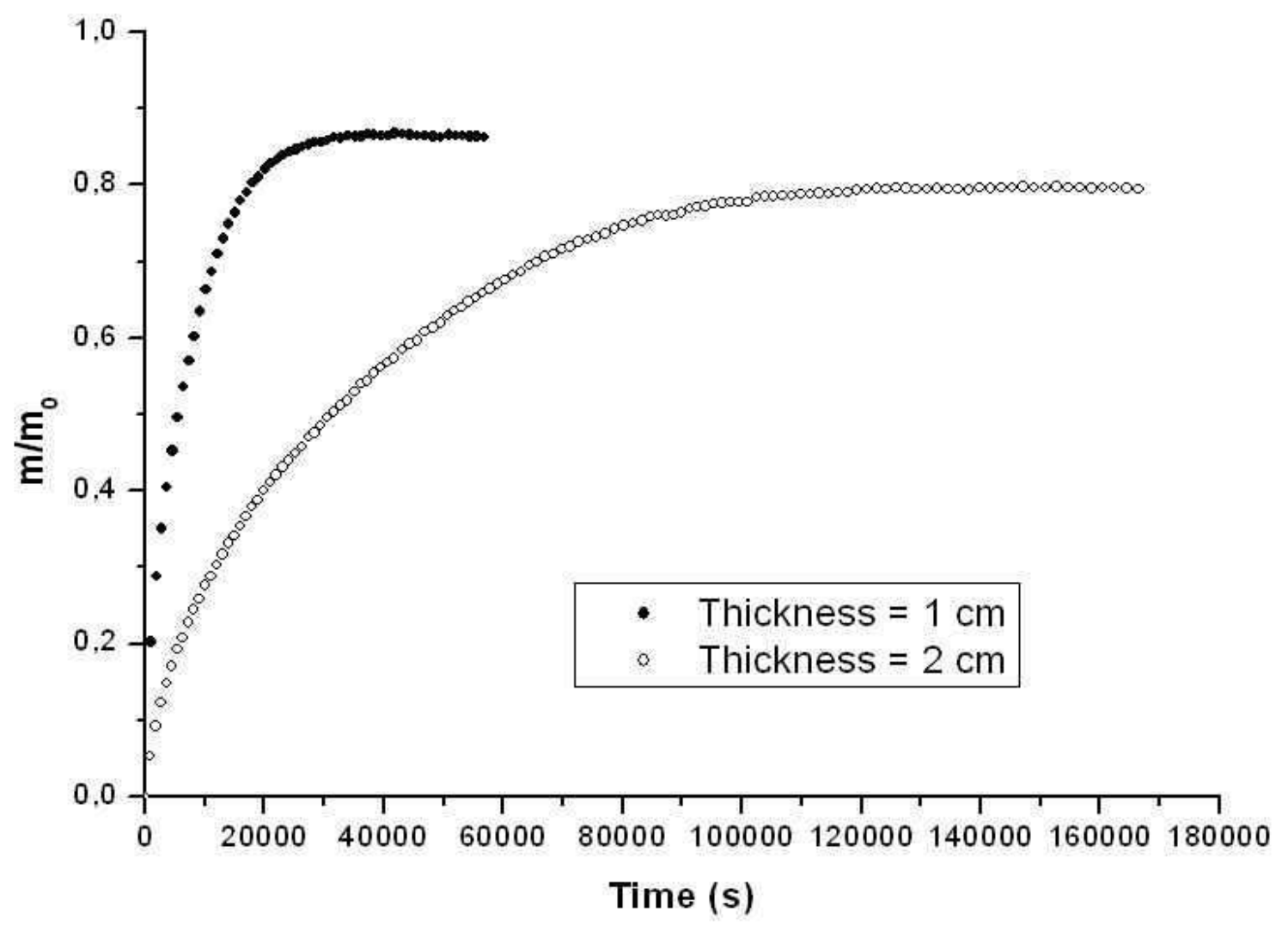


Figure 3:

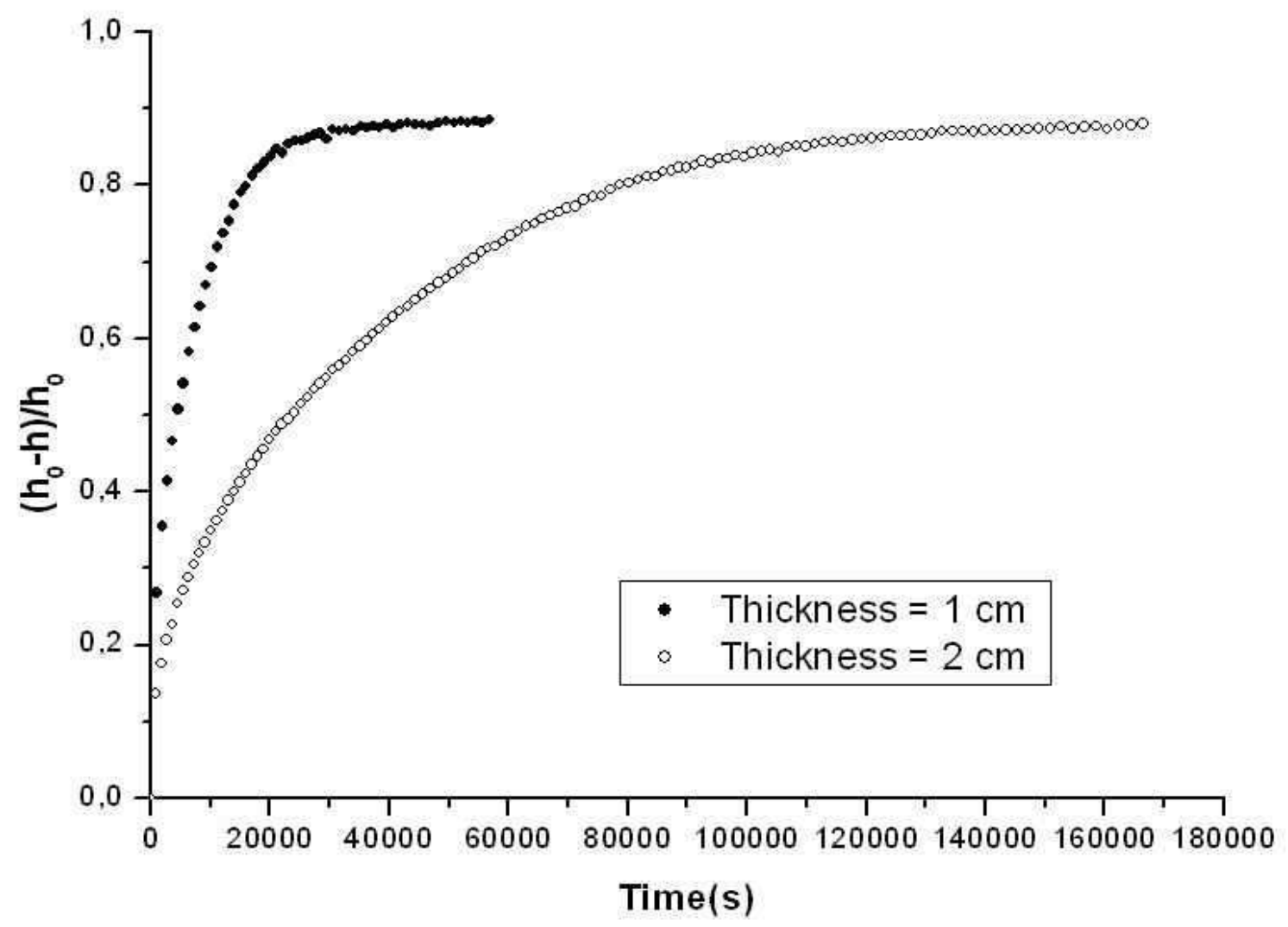


Figure 4:

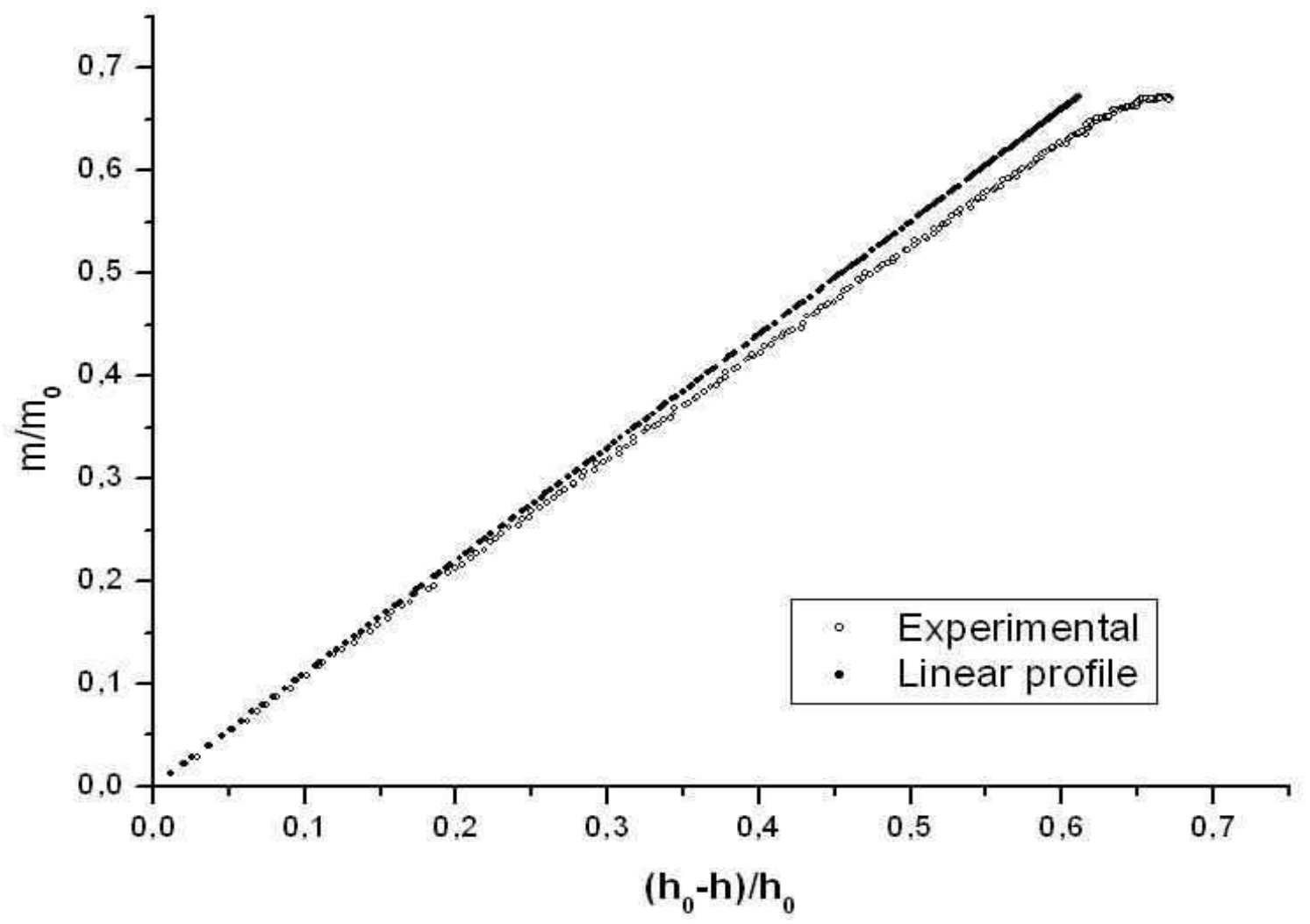


Figure 5:

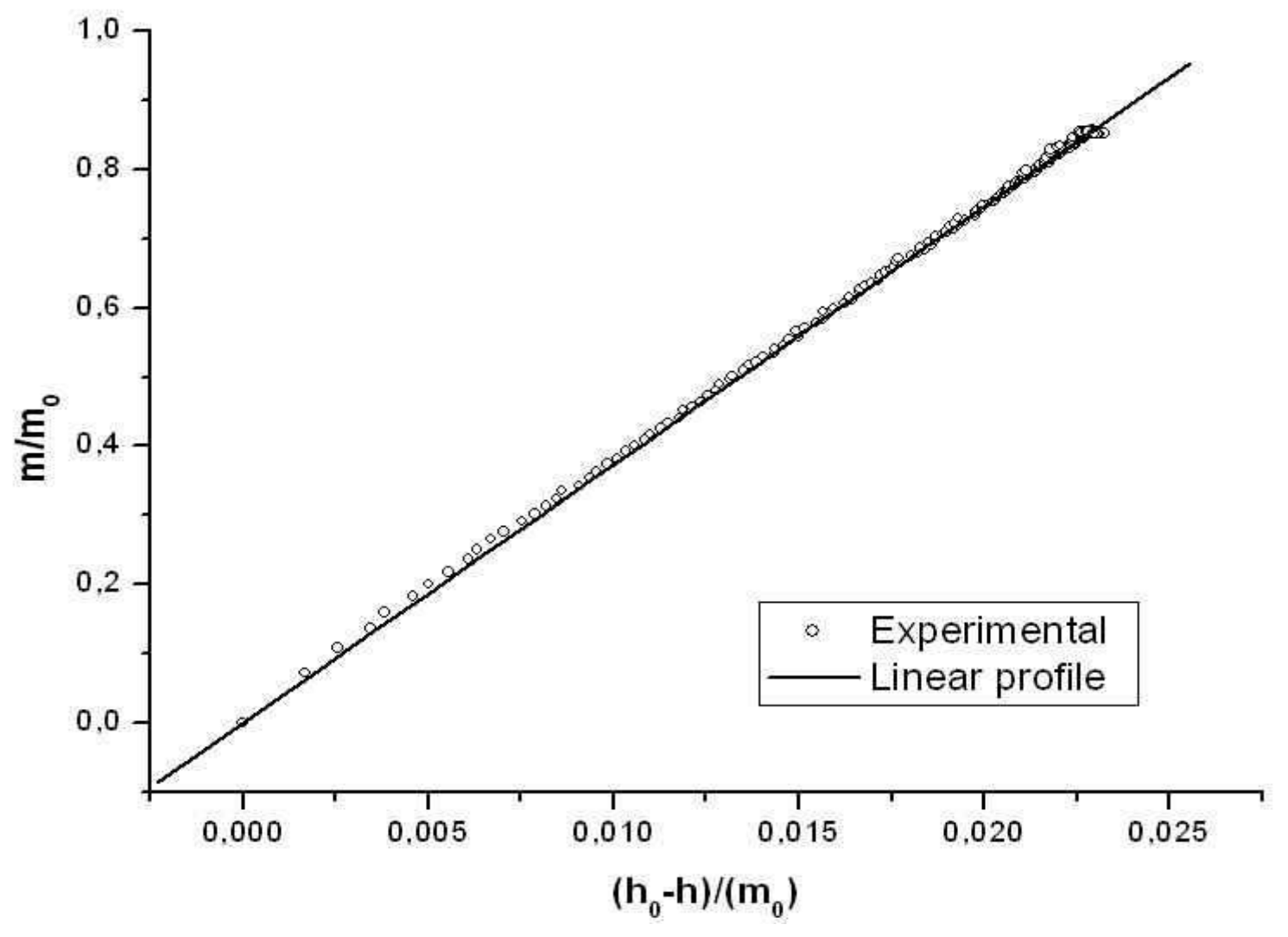


Figure 6:

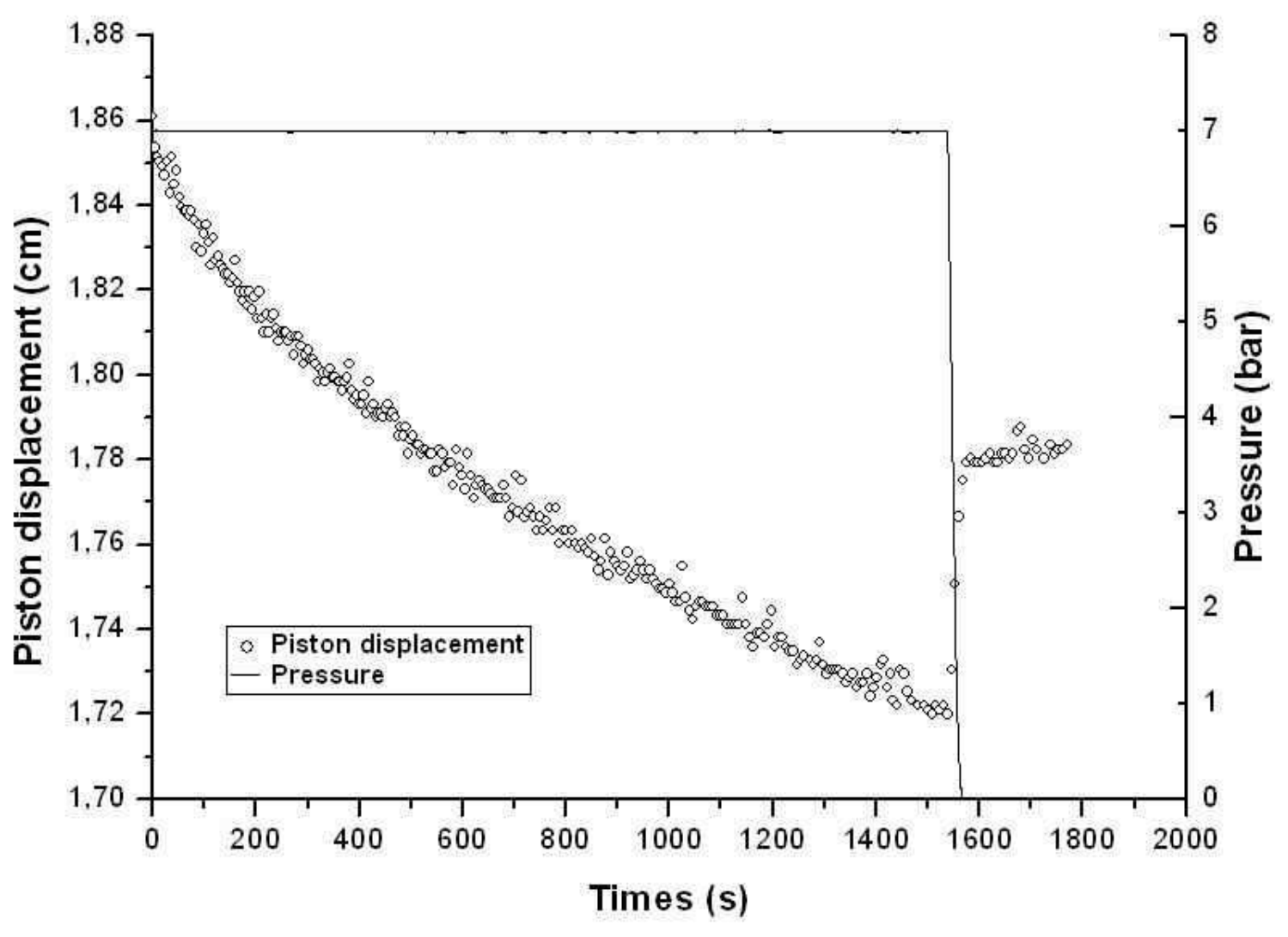


Figure 7:

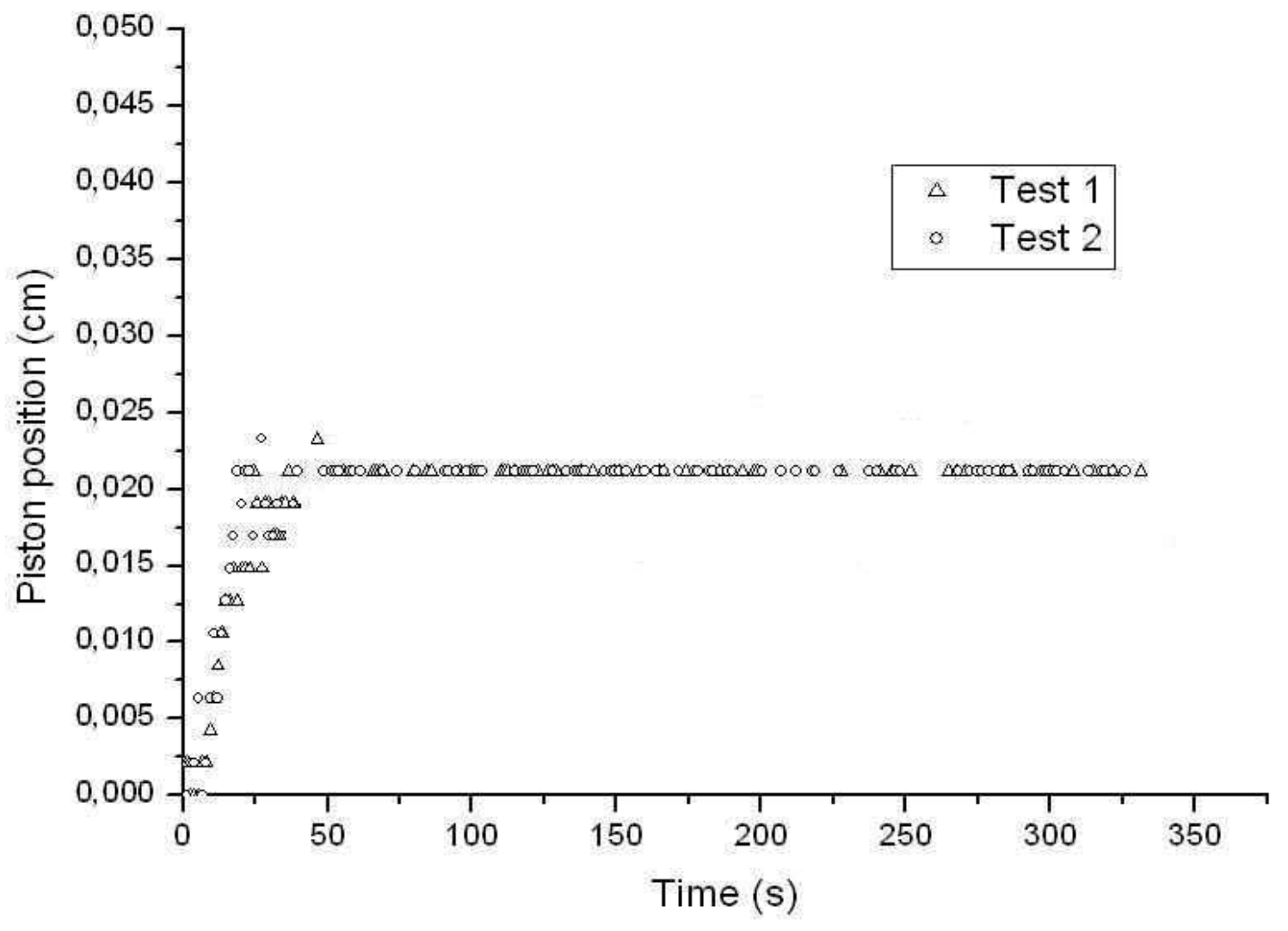


Figure 8:

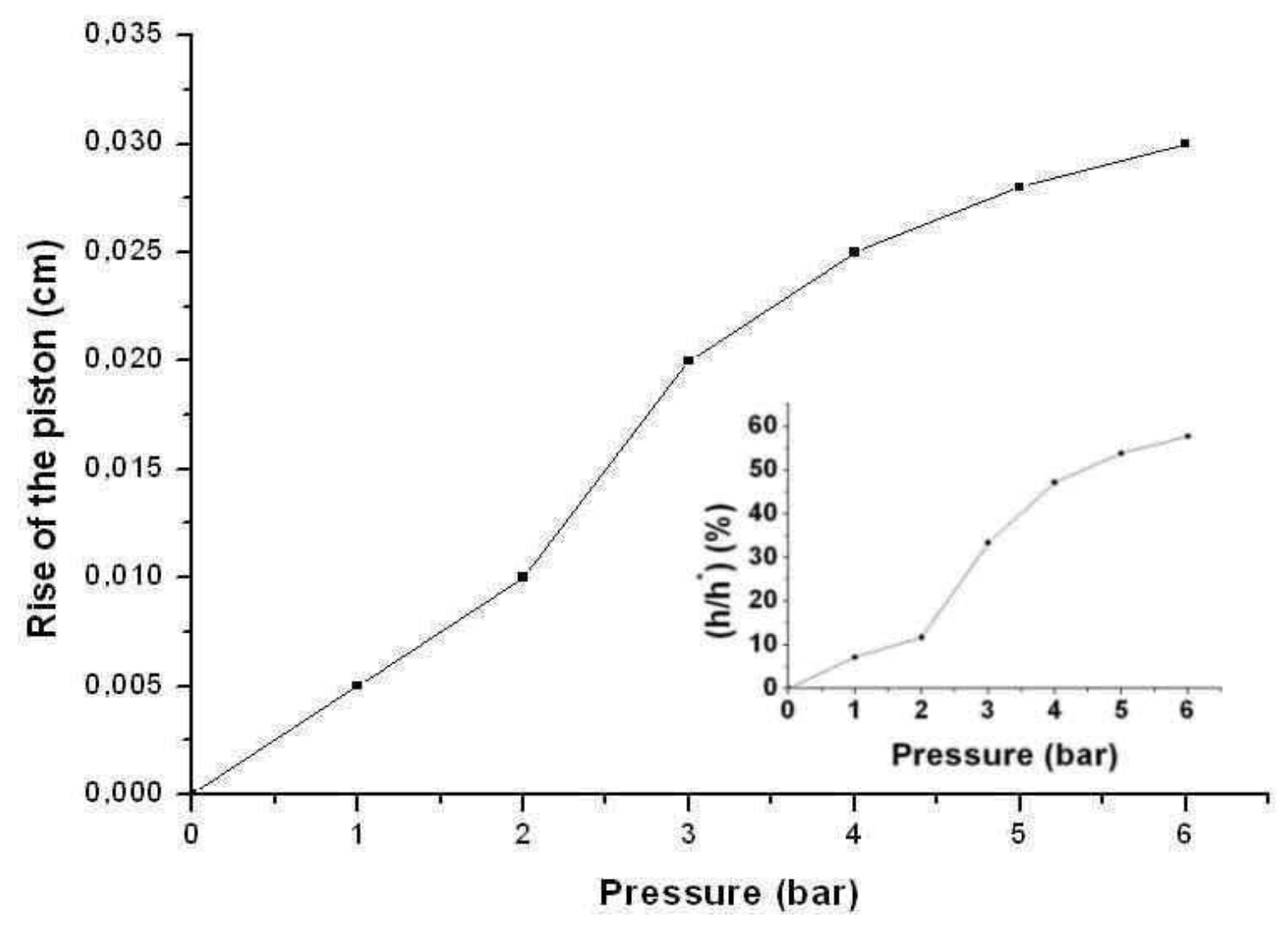


Figure 9:

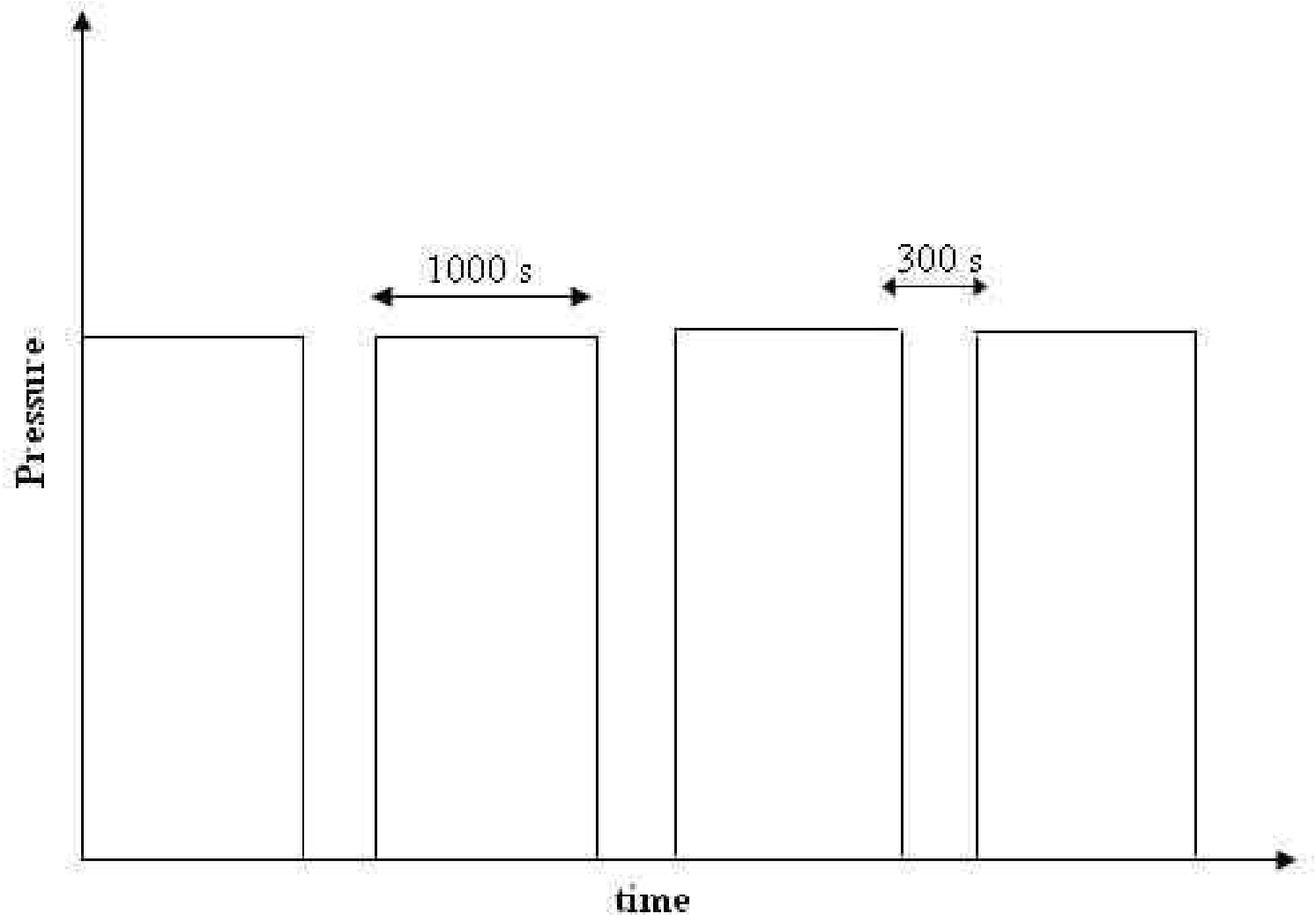


Figure 10:

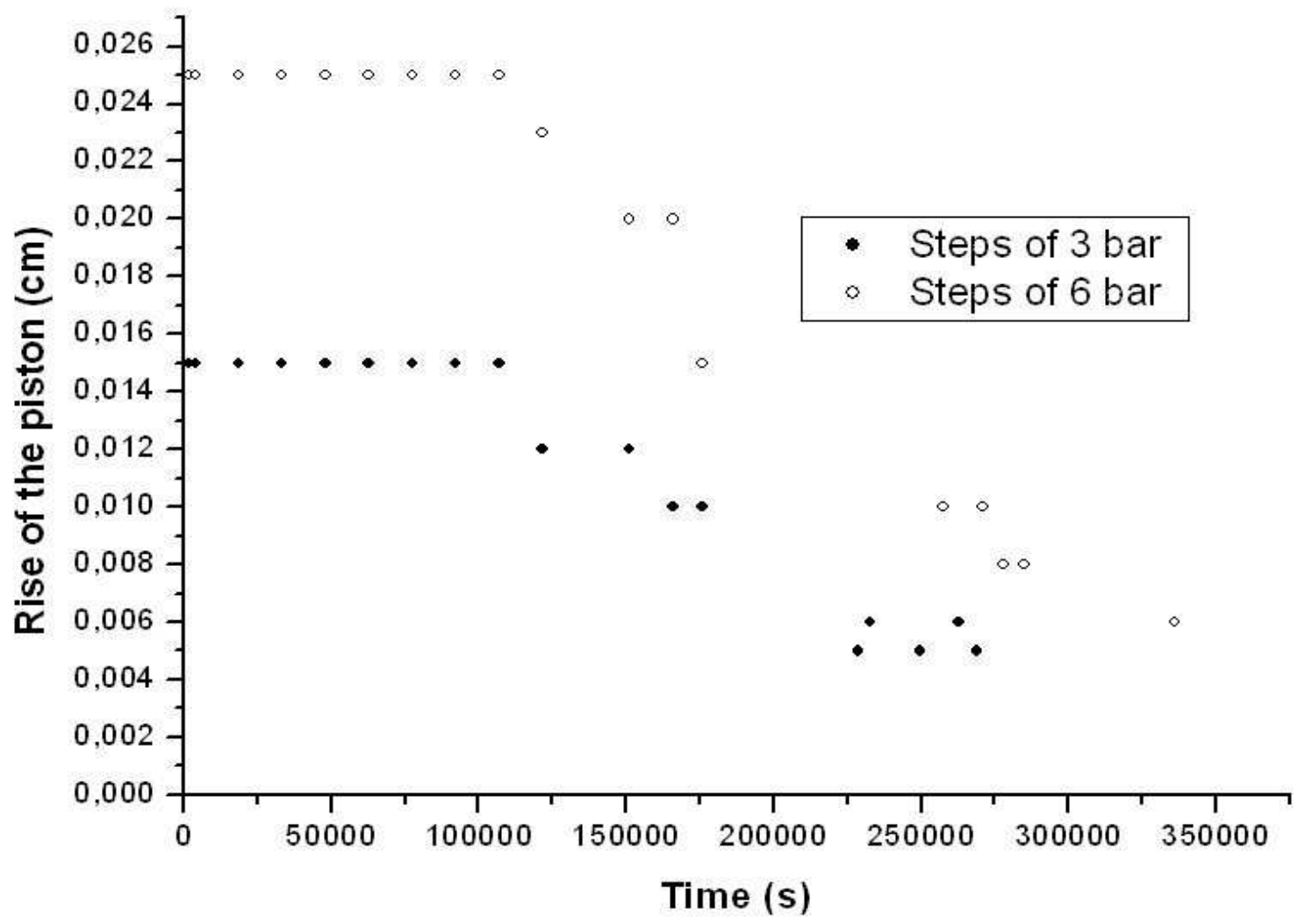




\section{Table captions}

Table 1: Rise of the piston versus time for 6 bar pressure steps (initial thickness of $2 \mathrm{~cm}$ ).

Table 2: Rise of the piston for different initial thicknesses of the sludge sample (6 bar pressure steps). 
Table 1:

\begin{tabular}{|c|c|c|c|}
\hline \multicolumn{2}{|c|}{ Semi-solid sludge } \\
\hline \multicolumn{2}{|c|}{ without salt } & with salt \\
\hline Dewatering time (s) & Rise of the piston (cm) & Dewatering time (s) & Rise of the piston (cm) \\
\hline 330 & 0.03 & 330 & 0.02 \\
790 & 0.03 & 790 & 0.02 \\
\hline 1200 & 0.03 & 1200 & 0.02 \\
\hline
\end{tabular}


Table 2:

\begin{tabular}{|c|c|}
\hline Initial thickness of sludge sample (cm) & Rise of the piston $(\mathbf{c m})$ \\
\hline 1 & 0.02 \\
\hline 2 & 0.02 \\
\hline 3 & 0.02 \\
\hline 4 & 0.02 \\
\hline 5 & 0.02 \\
\hline
\end{tabular}

